\title{
Design of a Small Modified Minkowski Fractal Antenna for Passive Deep Brain Stimulation Implants
}

\author{
Sara Manafi and Hai Deng \\ Department of Electrical and Computer Engineering, Florida International University, Miami, FL 33174, USA \\ Correspondence should be addressed to Sara Manafi; sara.manafi@gmail.com
}

Received 23 June 2014; Revised 7 August 2014; Accepted 1 September 2014; Published 4 November 2014

Academic Editor: Rashidul Islam

Copyright (C) 2014 S. Manafi and H. Deng. This is an open access article distributed under the Creative Commons Attribution License, which permits unrestricted use, distribution, and reproduction in any medium, provided the original work is properly cited.

A small planar modified Minkowski fractal antenna is designed and simulated in dual frequency bands (2.4 and $5.8 \mathrm{GHz})$ for wireless energy harvesting by deep brain stimulation (DBS) devices. The designed antenna, physically being confined inside a miniaturized structure, can efficiently convert the wireless signals in dual ISM frequency bands to the energy source to recharge the DBS battery or power the pulse generator directly. The performance metrics such as the return loss, the specific absorption rate (SAR), and the radiation pattern within skin and muscle-fat-skin tissues are evaluated for the designed antenna. The gain of the proposed antenna is $3.2 \mathrm{dBi}$ at $2.4 \mathrm{GHz}$ and $4.7 \mathrm{dBi}$ at $5.8 \mathrm{GHz}$; also the averaged SAR of the antenna in human body tissue is found to be well below the legally allowed limit at both frequency bands. The link budget shows the received power at the distance of $25 \mathrm{~cm}$ at $2.4 \mathrm{GHz}$ and $5.8 \mathrm{GHz}$ are around $0.4 \mathrm{~mW}$ and $0.04 \mathrm{~mW}$, which can empower the DBS implant. The large operational bandwidth, the physical compactness, and the efficiency in wireless signal reception make this antenna suitable in being used in implanted biomedical devices such as DBS pulse generators.

\section{Introduction}

Deep brain stimulation (DBS) is a surgical procedure using the implanted devices to send electrical simulation impulses to targeted areas in the brain to treat neurological disorders such as Parkinson's disease (PD) by blocking the abnormal nerve signals that could cause tremors or other PD symptoms $[1,2]$. A DBS system contains an implanted pulse generator (IPG) that is battery-powered and responsible for sending electrical pulses to brain to interfere with neural activity $[2,3]$. The average lifetime of IPG batteries is 5 to 7 years and a surgery is needed to replace the IPG batteries, which is very costly and inconvenient. In addition, the battery-powered implants lead to a larger physical size, making the further miniaturization of the implanted devices difficult, if not impossible. However, the battery issue could be solved by using passive batteryless or wireless rechargeable stimulation implants [4], which utilize an energy harvesting system converting natural or artificial electromagnetic (EM) waves to supplying power to either recharge the battery or directly power the implanted devices [5]. The electromagnetic sources used by an energy harvesting system could be the delicate source from the DBS external controller or random wireless sources such as mobile signals, wireless LANs, TV, and FM/ AM radio signals. A wireless EM-based energy harvesting system could be simply a receiving rectifying antenna, that is, rectenna, including an on-chip antenna and a rectifying circuit that converts the received sinusoids into a DC voltage. Therefore, if a rectenna or an energy harvesting system is implemented in the IPG of a DBS system, the DBS could be powered by random wireless sources that exist almost everywhere and all the time. The harvesting antenna is one of the most critical parts in a RF energy harvesting system, and designing harvesting antennas is especially challenging because it is directly related to the system efficiency and generally is application-specific. Hence, in this work we are focusing on the design of RF harvesting antenna specifically for passive DBS implants. A typical DBS system with an energy harvesting antenna is shown in Figure 1.

The designed harvesting antenna for the DBS should possess the following distinguishing features. (1) Compactness: the antenna is encased inside the IPG and its physical size 


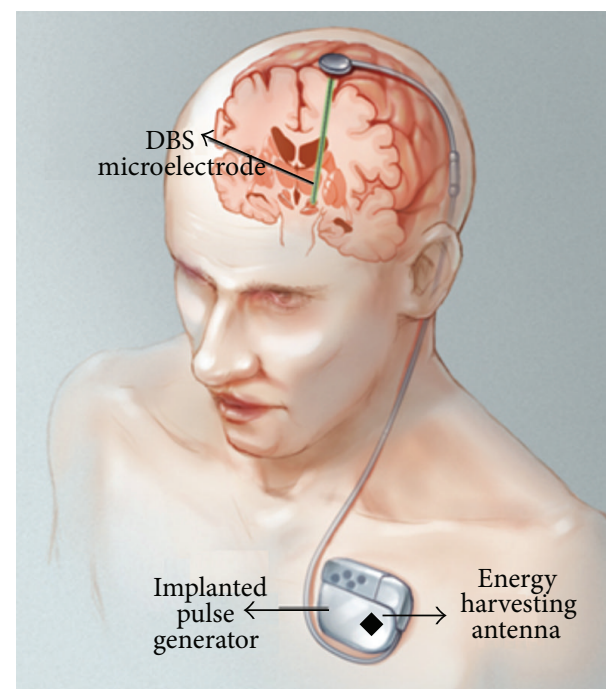

FIGURE 1: A typical DBS system with an energy harvesting antenna inside the implanted pulse generator (IPG).

is limited. (2) Readiness: the frequency and the bandwidth of the antenna must match the frequencies and bandwidths of opportunistic wireless signals that are widely available for energy harvesting. (3) Efficiency: the signal losses received by the antenna should be minimized. (4) Safety: the designed antenna must satisfy the government standards and regulations for safe application as implanted devices. Previously there were numerous reports on the design of implanted antennas [6-8]. However, the frequencies and bandwidths used by the existing implanted antennas are generally not suitable for RF energy harvesting with currently widely available WIFI or WiMAX signals.

In this work focusing on the antenna's characteristics is beneficial because it will boost the amount of harvestable energy. The broadband antenna receives relatively high RF power from various sources, and the accumulation of EM energy from various frequencies will increase the energy density of an EM wireless harvester. Hence, there is the need to have both multiband/broadband capabilities of the wireless EM harvester.

In addition, an antenna with space-filling self-similar fractal structure helps to generate multiple resonances with a compact size and wide bandwidth. In this work, we are set to design a new type of implanted DBS antennas based on Minkowski fractal strucutre because this structure is helpful to reduce the size of the proposed dual/wide band antenna for efficient energy harvesting in dual frequency bands at $2.4 \mathrm{GHz}$ and $5.8 \mathrm{GHz}$. With self-similar fractal structure, the size of the new antenna can be minimized for applications in implanted devices.

The new antenna is designed to operate in the dual frequencies in the industrial, scientific, and medical (ISM) radio bands with minimum losses, making the DBS systems highly efficient in RF energy harvesting with the ubiquitous WIFI and WiMAX signals.

\section{Antenna Design}

Normally for a typical DBS system, the size of an IPG is less than $47 \mathrm{~mm} \times 52 \mathrm{~mm} \times 11 \mathrm{~mm}$ and approximately half of the IPG space might be filled by the battery [9]. Therefore, we start the antenna design by limiting the size of the small modified Minkowski fractal antenna to a rectangular path antenna with a dimension of $20 \mathrm{~mm} \times 20 \mathrm{~mm} \times 1.6 \mathrm{~mm}$. Then a hybrid approach will be used to select and then refine the antenna parameters to improve its performance in energy harvesting under the physical constraints.

The simulation and performance properties such as return loss, gain, SAR, and radiation pattern are evaluated using the finite element method by HFSS software.

2.1. Initial Patch Antenna Selection. We choose Rogers $\mathrm{RO} 3210(\mathrm{tm})$ as the substrate for the antenna design with its thickness $h$ equal to $1.6 \mathrm{~mm}$. The relative dielectric constant $\varepsilon_{r}$ and the loss tangent $\delta$ of the material are 10.2 and 0.003 , respectively. Normally the materials with a high dielectric constant are preferred in the design of compact antennas. We can estimate the width, $W$, of the microstrip line using the transmission line theory [10]. Considering a square patch antenna initially, the length of the initial patch antenna, $L$, can be approximated with transmission line theory as well.

The side length $A$ of the square patch antenna is half of the $L$, since the radiation signal will be fed to the antenna through one of the corners, the length of the effective current flowing path is equal to the half of the square antenna perimeter; that is,

$$
L=2 A \text {. }
$$

The antenna's effective length is $23 \mathrm{~mm}$ at $2.4 \mathrm{GHz}$ and $9.6 \mathrm{~mm}$ at $5.8 \mathrm{GHz}$ in free space; thus the initial size length $A$ of the patch antenna is equal to about $12 \mathrm{~mm}$. However, if the antenna is implanted inside the human body as a part of IPG, the actual resonant frequency would be shifted to a lower frequency due to the higher relative dielectric constant of body tissues. Hence, the implanted antenna should be designed at higher resonant frequencies in free space. Consequently the effective antenna length should be shorter than the result obtained using the transmission line theory.

2.2. Minkowski Iterations and Additional Modifications. To make the antenna efficient in dual frequency bands, we apply the Minkowski fractal miniaturization technique to the square patch to increase the length of the current flowing path and thus decrease the antenna physical size. The basic Minkowski fractal operator, shown in Figure 2(a), is used to increase the effective current flowing lengths from $A$ to $A+2 C$ with $C$ defined as the fractal depth. The Minkowski fractal operator could be repetitively applied to each segment generated from the Minkowski fractal operation at previous iteration level to further increase the current flowing path lengths and minimize the antenna size for a particular resonant frequency.

Figure 2(b) shows the results of applying the Minkowski fractal operator to each side of the square patch antenna in 


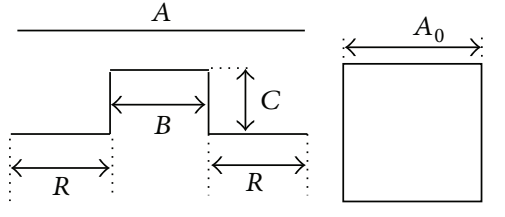

(a)

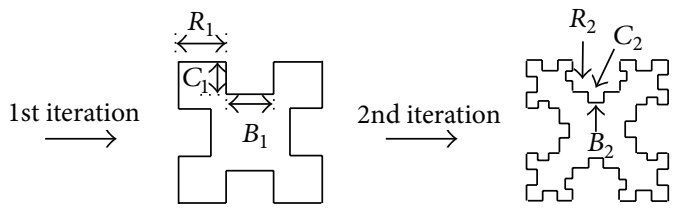

(b)

Figure 2: (a) The Minkowski fractal operator and (b) the first and second Minkowski fractal iterations of a square patch antenna.

two iterations. Generally by using the Minkowski method one can perform $n$th iteration by dividing a straight segment $A_{n-1}$ into five subsegments $R_{n}-C_{n}-B_{n}-C_{n}-R_{n}$ with the following constraint:

$$
A_{n-1}=2 R_{n}+B_{n}
$$

where $C_{n}$ is the fractal depth in the $n$th iteration of the Minkowski algorithm and $\eta_{n}$ is the iteration factor in the iteration and is defined as

$$
\eta_{n}=\frac{C_{n}}{B_{n}} .
$$

The values of both $C_{n}$ and $\eta_{n}$ at the $n$th iteration are adjustable and can be optimized based on the performance of the formed antenna. In this work, we optimize the parameters based on the total return loss at the resonant frequencies.

The Minkowski fractal antennas obtained from the first and second iterations of the square patch antenna with a side length of $12 \mathrm{~mm}$ are shown in Figures 3(a) and 3(b). With the first Minkowski iteration, the resonant frequency of the antenna is found to be about $6.2 \mathrm{GHz}$, while the second iteration generates two resonant frequencies at about 3.1 GHz and $5.8 \mathrm{GHz}$. The coupling between the sharp angles of the Minkowski fractal structure produces different current paths making dual frequency band operation possible. The Minkowski fractal antenna structure shifts the resonant frequency slightly downward due to the longer current paths generated from the iterations.

In this design, the antenna needs to resonate at $2.4 \mathrm{GHz}$ and $5.8 \mathrm{~Hz}$ for dual band harvesting. To generate resonance at $2.4 \mathrm{GHz}$, the antenna should have an effective length of about $20 \mathrm{~mm}$. Because the side length of the initial square patch antenna is $12 \mathrm{~mm}$, the equivalent antenna length of the Minkowski fractal antenna after two iterations is estimated to be about $D \times 12 \mathrm{~mm}$, where $D$ is the fractal dimension extension factor. Based on the Hausdorff-Besicovitch equation [11], $D$ is about 1.5 and leads to the equivalent antenna length of about $18 \mathrm{~mm}$, which is not sufficient for generating a resonance frequency at $2.4 \mathrm{GHz}$. However, further increasing the number of Minkowski fractal iterations generally would not be able to generate the intended resonant frequency and also could make the manufacturing of the designed antenna technically challenging. In order to extend the effective antenna sizes, we modify the Minkowski fractal structure in Figure 3(b) by adding the scaled-down replicas of the antenna itself to the three open corners of the antenna. The structure of the modified Minkowski fractal antenna is shown in Figure 3(c). The total structure is still confined inside a dimension of $20 \mathrm{~mm} \times 20 \mathrm{~mm}$. The modified antenna can provide larger current paths in operation and thus makes it possible to generate a resonant frequency at $2.4 \mathrm{GHz}$. However, the parameters such as iteration factors and fractal depths of the modified Minkowski fractal antenna could be further optimized based on the return loss to achieve the best $\mathrm{RF}$ energy harvesting results in the dual frequency bands.

Besides the radiating part and the microstrip feed line in Figure 3(c), this antenna also contains two passive triangle elements and a defected ground plane, shown as the bottom layer in Figure 3(d). The defected ground plane is advantageous in expanding the antenna bandwidth and provides better performance than the usual antenna patches [12]. The two passive elements in the radiating layer enable the antenna to be better matched to the $50-\Omega$ feed line. They also serve a role in exciting additional resonant modes to widen the system bandwidth.

2.3. Parameters Optimization. The patch antenna preliminarily designed in the last section includes the basic part generated directly from the first and second Minkowski fractal iterations and the three identical scaled-down replicas of the basic part. Therefore, the parameters of the Minkowski fractal iterations such as iteration factors $\eta$ and fractal depths $C$ for both basic part and replicas can be optimized for minimizing the total return loss in two resonant frequencies. In this design, the iteration factors and the fractal depths of the first and second iterations for the basic part in Figure $3(\mathrm{c})$ are assumed to be $\eta_{1}, \eta_{2}, C_{1}$, and $C_{2}$, respectively; and similarly for the replicas the four parameters are defined as $\eta_{1}^{*}, \eta_{2}^{*}, C_{1}^{*}$, and $C_{2}^{*}$, respectively. To simplify the optimization, we limit the parameter selection on a grid; that is, $\eta_{1}$ changes with a step size of 0.1 and the initial value at 1 during the optimization; and $\eta_{1}^{*}, \eta_{2}$, and $\eta_{2}^{*}$ with a step size of 0.05 and the initial value at 0.1 . In addition, we separate the optimization of the basic part from that of the replicas to reduce the optimization time. The cost function of the optimization is the total return loss at frequencies of $2.4 \mathrm{GHz}$ and $5.8 \mathrm{GHz}$. We use a simple yet efficient relaxation algorithm to optimize the antenna parameters. The procedures of optimizing $\eta_{1}, \eta_{2}, C_{1}$, and $C_{2}$ for the basic part in the first and second Minkowski iterations are detailed in the following steps.

Step 1. Start by selecting initial values for $\eta_{1}, \eta_{2}, C_{1}$, and $C_{2}$.

Step 2. Incrementally increase the value of $\eta_{1}$ by a step size, evaluate the cost function at each step with other parameters fixed, and pick the value for $\eta_{1}$ with the minimum cost. 


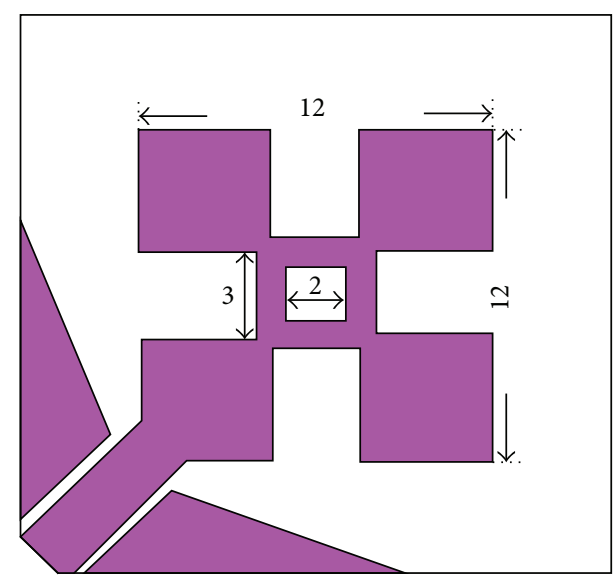

(a)

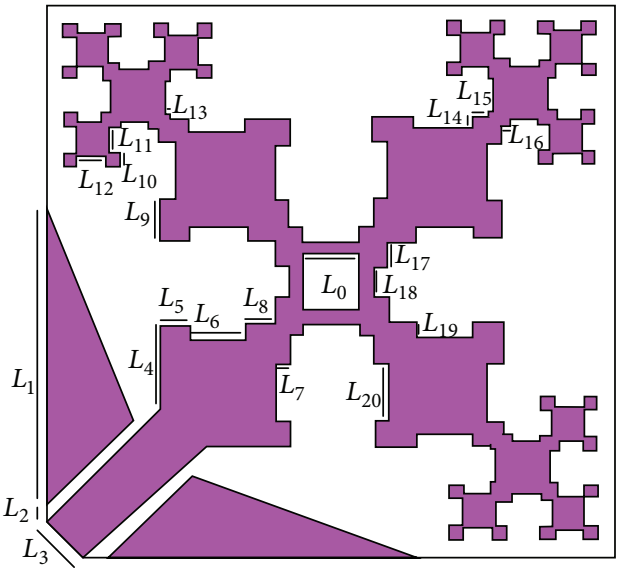

(c)

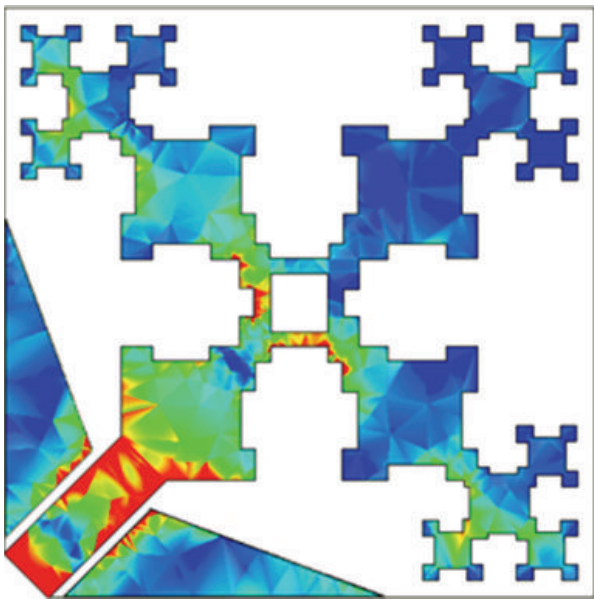

(e)
$J_{\text {surf }}(\mathrm{A} / \mathrm{m})$

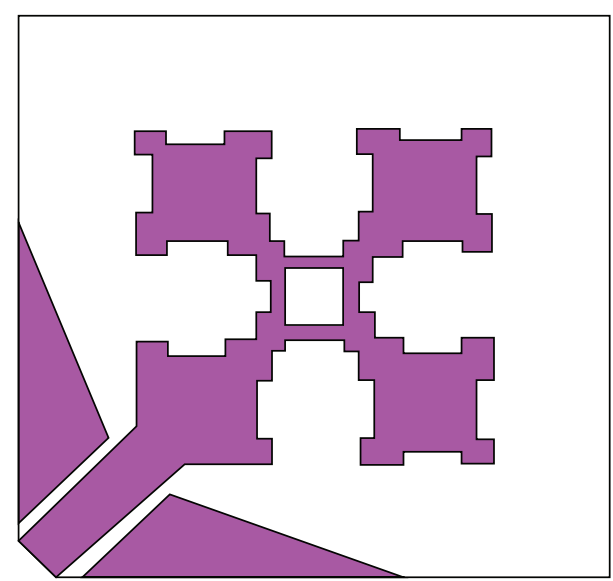

(b)

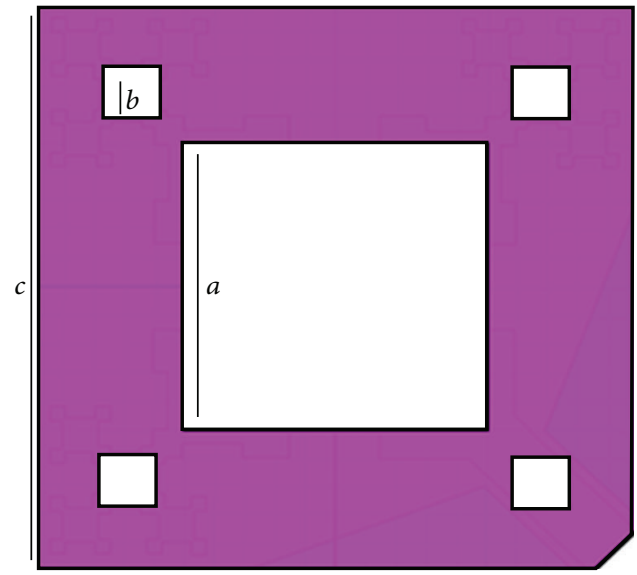

(d)
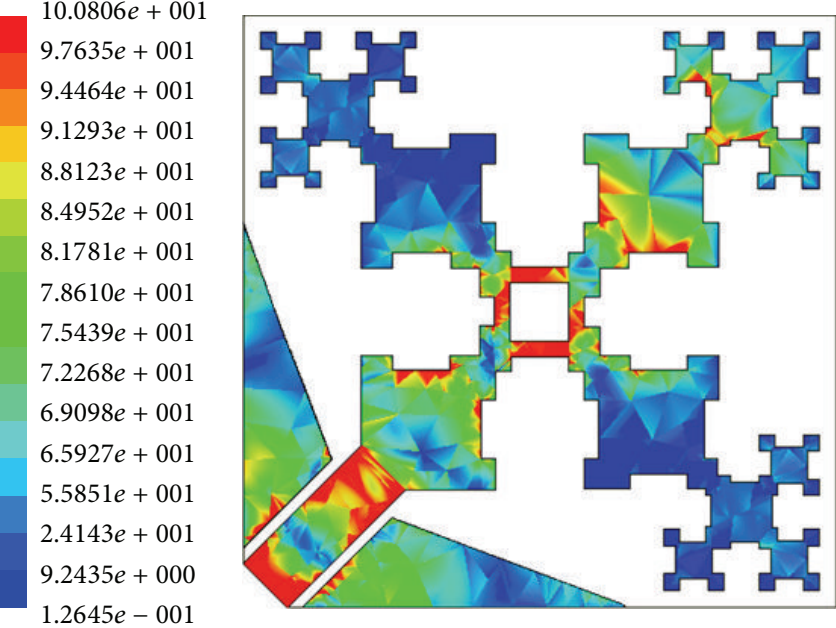

(f)

FIGURE 3: Geometries of the Minkowski fractal antennas (a) in first iteration and (b) in the second iteration (measured in mm), and the geometry of the optimized Minkowski fractal antenna shown on (c) top layer and (d) bottom layer. The designed antenna's current distribution at (e) $2.4 \mathrm{GHz}$ and (f) $5.8 \mathrm{GHz}$. 


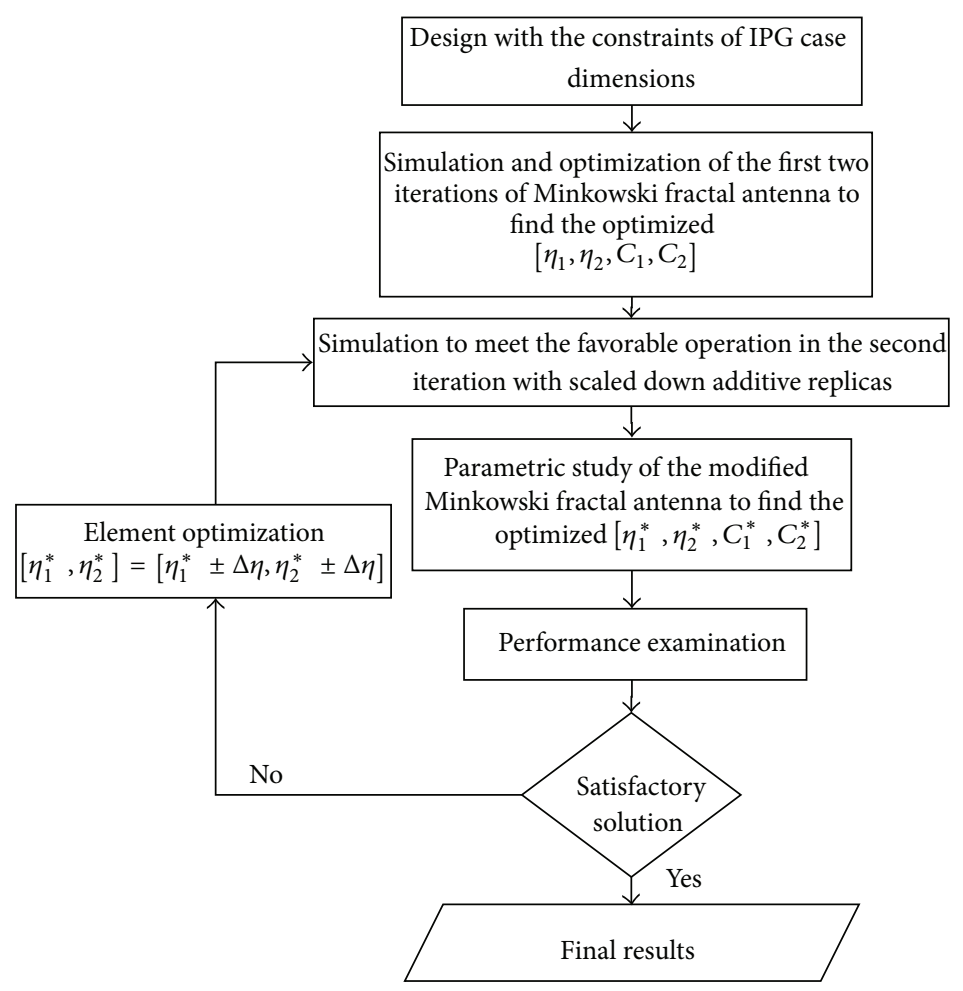

FIGURE 4: The flowchart of designing and parameter optimization process of the proposed modified Minkowski fractal antenna.

Step 3. Repeat Step 2 with each of parameters $\eta_{2}, C_{1}$, and $C_{2}$, separately.

Step 4. Repeat Steps 2-3 until the cost cannot be reduced.

Step 5. Stop.

Having a smooth and wider return loss after optimization, $\eta_{1}$ equals 1.2 and $\eta_{2}$ is equal to 0.25 . The final parameter values obtained in Step 5 are the optimal $\eta_{1}, \eta_{2}, C_{1}$, and $C_{2}$ optimized for the basic part of the antenna. The optimal values of $L_{4}, L_{5}, L_{6}, L_{7}, L_{8}, L_{9}, L_{14}, L_{15}, L_{17}, L_{18}, L_{19}$, and $L_{20}$ in Figure 3(c) can be found from the optimized $\eta_{1}, \eta_{2}, C_{1}$, and $C_{2}$ accordingly. Similarly the parameters for the antenna replicas are optimized with the same relaxation algorithm. Subsequently, the optimal values of $L_{10}, L_{11}, L_{12}, L_{13}$, and $L_{16}$ in Figure 3(c) can be calculated from the optimal $\eta_{1}^{*}, \eta_{2}^{*}$, $C_{1}^{*}$, and $C_{2}^{*}$; these values are listed below: $a=10 \mathrm{~mm}, b=$ $1.5 \mathrm{~mm}, c=20 \mathrm{~mm}, L_{0}=2 \mathrm{~mm}, L_{1}=11 \mathrm{~mm}, L_{2}=0.5 \mathrm{~mm}$, $L_{3}=2.1 \mathrm{~mm}, L_{4}=3 \mathrm{~mm}, L_{5}=1 \mathrm{~mm}, L_{6}=2 \mathrm{~mm}$, $L_{7}=0.5 \mathrm{~mm}, L_{8}=1 \mathrm{~mm}, L_{9}=1.5 \mathrm{~mm}, L_{10}=0.5 \mathrm{~mm}$, $L_{11}=1 \mathrm{~mm}, L_{12}=1 \mathrm{~mm}, L_{13}=0.2 \mathrm{~mm}, L_{14}=0.5 \mathrm{~mm}$, $L_{15}=0.6 \mathrm{~mm}, L_{16}=0.4 \mathrm{~mm}, L_{17}=1 \mathrm{~mm}, L_{18}=1 \mathrm{~mm}$, $L_{19}=0.5 \mathrm{~mm}, L_{20}=2 \mathrm{~mm}$.

After optimization $\eta_{1}^{*}$ equals 1.4 and $\eta_{2}^{*}$ is equal to 0.5 . The designing and step by step parameter optimization process of the proposed antenna is summarized in the flowchart that is shown in Figure 4.

To verify the resonance frequencies of the designed antenna at $2.4 \mathrm{GHz}$ and $5.8 \mathrm{GHz}$, we simulated the current distributions of this antenna. The current distributions for those two frequencies are shown in Figures 3(e) and 3(f), respectively. The current in Figure 3(e) shows the half-wavelength paths formed from the center of the antenna to its upper-left and lower-right corners at $2.4 \mathrm{GHz}$. Figure 3(f) shows the current paths formed in the lower-left and upperright patches in the basic part of the antenna for the feeding source at $5.8 \mathrm{GHz}$.

\section{Simulation Results}

For the implanted antenna in DBS applications, the antenna operates inside the human body in the chest area with various biological tissues as the possible EM wave propagation medium. The antenna is optimized for the minimum return loss in the intended resonant frequencies inside the chest. Therefore, we need to further evaluate the designed antenna performance such as return loss in human tissues. The likely human tissues include skin, muscle, and fat and their electrical parameters such as $\varepsilon$ and $\sigma$ are different and frequency-dependent.

Depending on the location of the implanted antenna, its operational medium could be modeled as one-layer skin, as shown in Figure 5(a) or the three-layer combination of muscle-fat-skin in Figure 5(b) for performance evaluation [13]. The parameters such as permittivity, conductivity, and mass density for those biological tissues used in the simulation are summarized in Table 1 [14].

Figure 6(a) shows the frequency-domain return loss $\left(S_{11}\right)$ of the designed antenna (Figure 3(c)) in two different types of biological tissues. For comparison, the return losses for 
TABle 1: The parameters of the biological tissues at $2.4 \mathrm{GHz}$ and $5.8 \mathrm{GHz}$ [14].

\begin{tabular}{|c|c|c|c|c|c|c|}
\hline Tissue & \multicolumn{2}{|c|}{ Skin } & \multicolumn{2}{|c|}{ Fat } & \multicolumn{2}{|c|}{ Muscle } \\
\hline Frequency $(\mathrm{GHz})$ & 2.4 & 5.8 & 2.4 & 5.8 & 2.4 & 5.8 \\
\hline Permittivity & 38.1 & 35.1 & 10.8 & 9.86 & 52.8 & 48.5 \\
\hline Conductivity & $1.44 \mathrm{~s} / \mathrm{m}$ & $3.72 \mathrm{~s} / \mathrm{m}$ & $0.26 \mathrm{~s} / \mathrm{m}$ & $0.83 \mathrm{~s} / \mathrm{m}$ & $1.71 \mathrm{~s} / \mathrm{m}$ & $4.96 \mathrm{~s} / \mathrm{m}$ \\
\hline Mass density & \multicolumn{2}{|c|}{$1080 \mathrm{~kg} / \mathrm{m}^{3}$} & \multicolumn{2}{|c|}{$920 \mathrm{~kg} / \mathrm{m}^{3}$} & \multicolumn{2}{|c|}{$1050 \mathrm{~kg} / \mathrm{m}^{3}$} \\
\hline
\end{tabular}

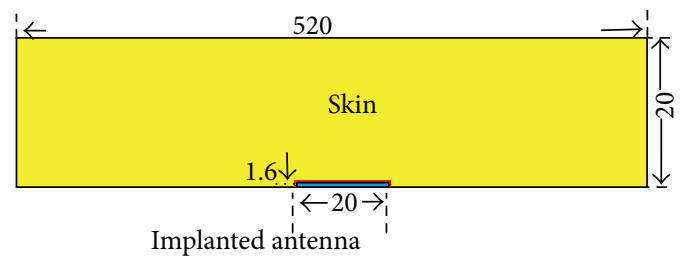

(a)

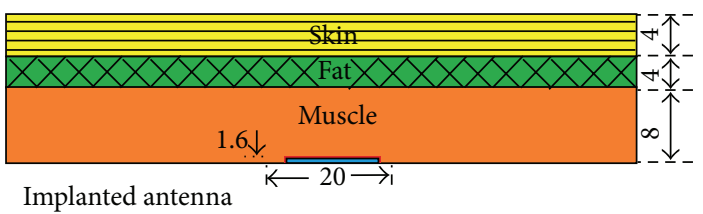

(b)

FIGURE 5: The designed antenna implanted in (a) skin tissue and (b) muscle-fat-skin tissues (measured in mm).

the standard Minkowski antennas with the first and second iterations, that is, the basic part of the designed antenna in Figures 3(a)-3(b) without the additive replicas, are also plotted in the same figure. The simulation results indicate that two resonant frequencies are located at about $2.6 \mathrm{GHz}$ and $5.2 \mathrm{GHz}$ for the designed antenna operating in human tissues. However, the return loss at $2.4 \mathrm{GHz}$ or $5.8 \mathrm{GHz}$ is 1$3 \mathrm{~dB}$ larger than the minimum value, which demonstrates a large energy harvesting bandwidth for the designed antenna. Furthermore, the input impedance of the antenna is found to be $Z_{i}=46.735+j 0.0542$ at $2.4 \mathrm{GHz}$ and $Z_{i}=40.849+0.0683 j$ at $5.8 \mathrm{GHz}$; hence, the antenna has near zero reactance around $2.4 \mathrm{GHz}$ and $5.8 \mathrm{GHz}$. From Figure $6(\mathrm{a})$, one can find the standard Minkowski antenna with the first or second iteration incurs much larger return losses at $2.4 \mathrm{GHz}$ and $5.8 \mathrm{GHz}$ with much narrowed bandwidth.

The radiation beam patterns of the designed antenna are computed for operating in muscle-fat-skin tissues at $2.4 \mathrm{GHz}$ and $5.8 \mathrm{GHz}$ and the results are displayed in Figures 6(b)6(e). The maximum gain for the implanted antenna is found to be $3.2 \mathrm{dBi}$ at $2.4 \mathrm{GHz}$ and $4.7 \mathrm{dBi}$ at $5.8 \mathrm{GHz}$ which are close to the free space gain that equals $4.1 \mathrm{dBi}$ at $2.4 \mathrm{GHz}$ and $5.9 \mathrm{dBi}$ at $5.8 \mathrm{GHz}$. The radiation pattern of designed antenna in the body issue is almost omnidirectional, which allows efficient $\mathrm{RF}$ energy harvesting in almost any direction for the external random radiation sources.

The specific absorption rate (SAR) is used to measure the RF power absorbed by the human tissues within a unit volume due to the implanted antenna. In rectenna the RF electrical current induces electric field in surrounding tissues, and due to the lossy medium of human body tissues some part of the far filed radiated EM wave from transmitter will be absorbed by biological tissues of human body as well. The SAR inside the body tissues is defined by

$$
\operatorname{SAR}=\frac{\sigma}{2 \rho}\left|E_{t}\right|^{2}
$$

where $E(\mathrm{~V} / \mathrm{m})$ is the electric field intensity in the tissue and $\sigma(\mathrm{S} / \mathrm{m})$ and $\rho\left(\mathrm{kg} / \mathrm{m}^{3}\right)$ are the conductivity and the density of the human tissue, respectively. Figures 6(f)-6(g) show the averaged SAR of the designed antenna in the human chest with the dimensions of $520 \mathrm{~mm} \times 400 \mathrm{~mm} \times 20 \mathrm{~mm}$ at $2.4 \mathrm{GHz}$ and $5.8 \mathrm{GHz}$, respectively. Based on the harvested power (in Table 2) the maximum induced SAR at $2.4 \mathrm{GHz}$ and $5.8 \mathrm{GHz}$ are about $0.4 \mathrm{~W} / \mathrm{Kg}$ and $0.04 \mathrm{~W} / \mathrm{Kg}$. Since the maximum legal SAR level allowed for implanted devices is $1.6 \mathrm{w} / \mathrm{kg}$ [15], the simulation results in Figures 6(f)-6(g) show that the SAR of the antenna is well below the acceptable level. Therefore, the designed antenna is safe for being implanted in human body.

It should be noted that the encapsulation material for the antenna in the simulation is generally assumed to be silicon rubber with its thickness equal to $0.5 \mathrm{~mm}$. The dielectric constant of silicon rubber has a wide range of 3.2-9.8 [16], but in this work it is considered with 3.2 dielectric constant and 0.01 loss tangent [17]. Therefore, it would almost have no effect on the impedance or radiation pattern of the implanted antenna [18].

\section{Energy Harvesting Performance Evaluation}

The designed antenna is to be implanted in human chest to harvest RF energy from an external radiation source such as 


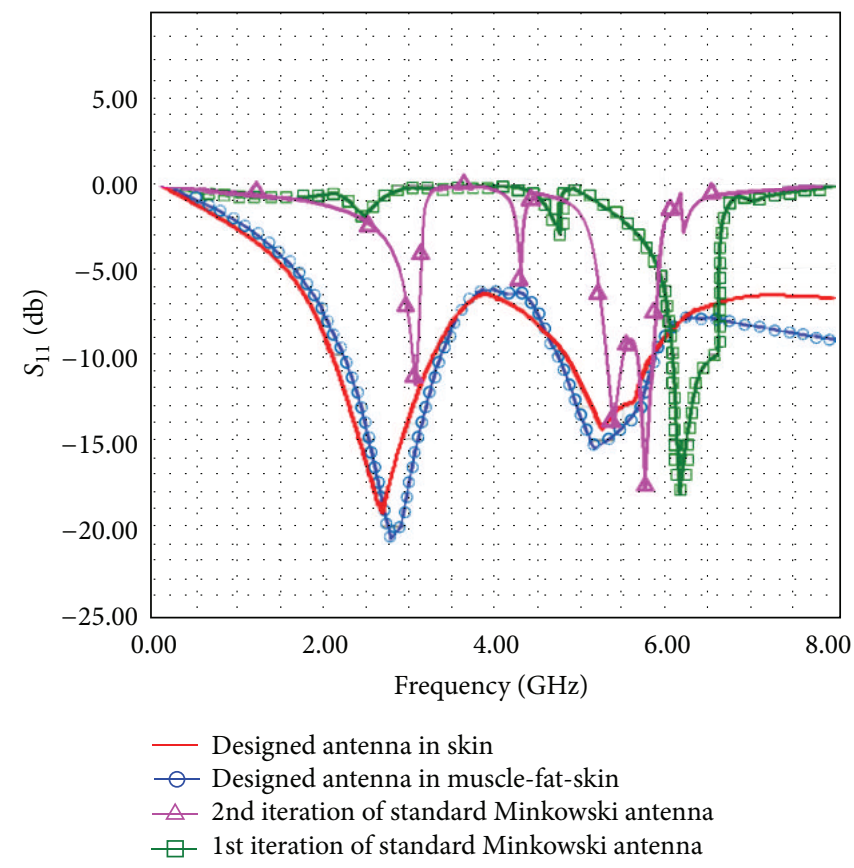

(a)

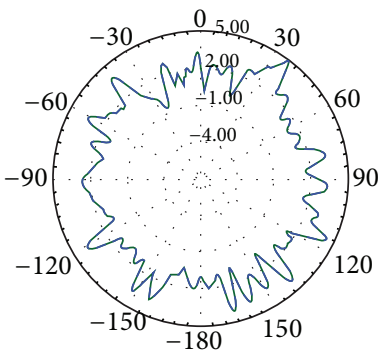

(b)

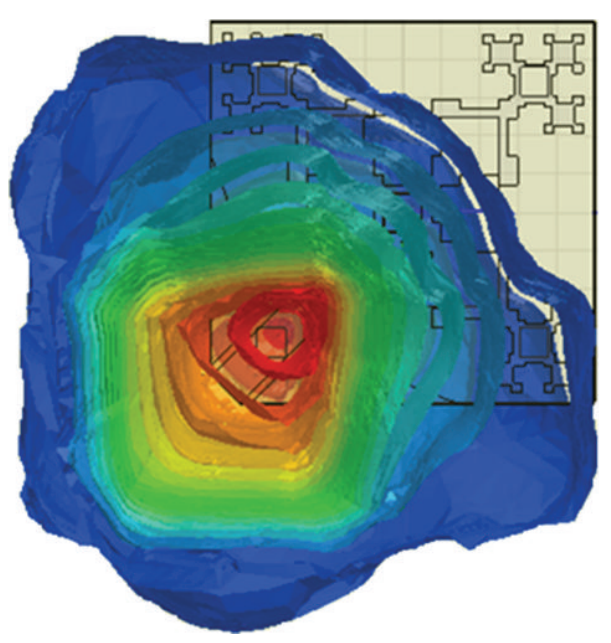

(f)

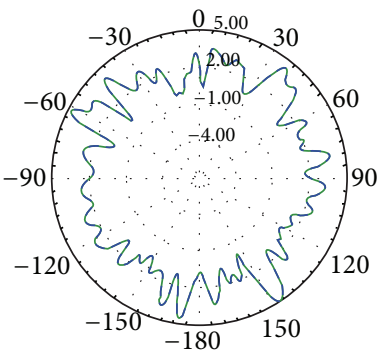

(c)

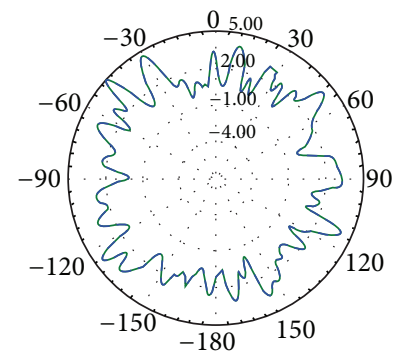

(d)

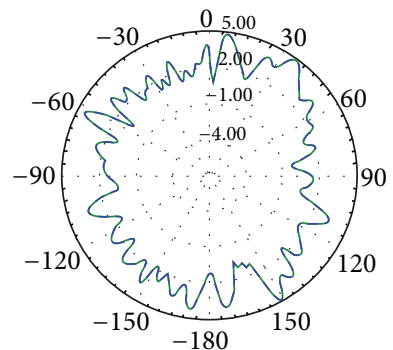

(e)

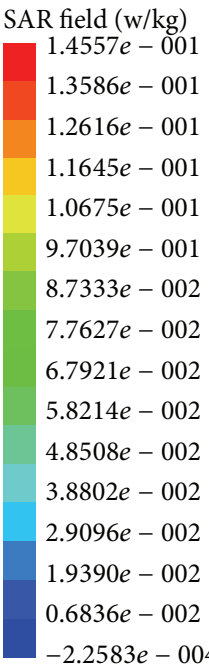

$-2.2583 e-004$

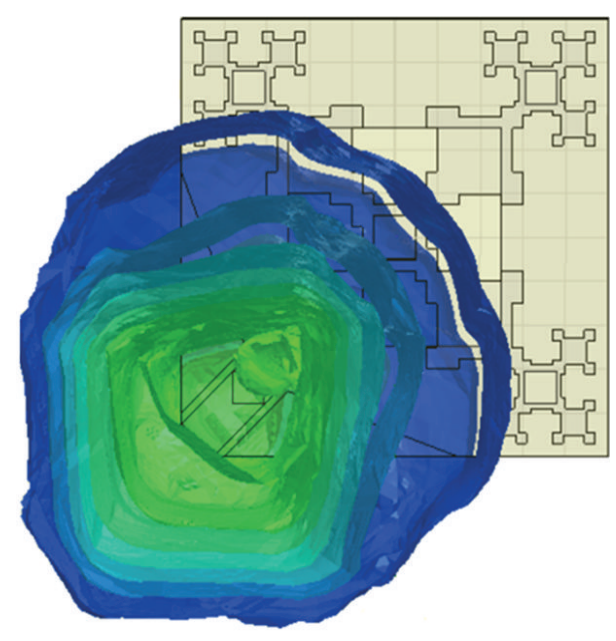

(g)

Figure 6: (a) The return losses $\left(S_{11}\right)$ of the designed antenna in human body and the 1st and 2nd iterations of standard Minkowski fractal antenna in skin. (b)-(e) The Gain patterns of the designed antenna plotted in azimuth plane $(\theta=90)$ at (b) $2.4 \mathrm{GHz}$ and (c) $5.8 \mathrm{GHz}$ and in zenith plane $(\varphi=90)$ at (d) $2.4 \mathrm{GHz}$ and (e) $5.8 \mathrm{GHz}$. The total SAR from the top view at (f) $2.4 \mathrm{GHz}$ and (g) $5.8 \mathrm{GHz}$ inside the human body. 
TABle 2: Parameters and results for performance evaluation tests.

\begin{tabular}{lcc}
\hline Parameters of link budget & Frequency $(2.4 \mathrm{GHz})$ & Frequency $(5.8 \mathrm{GHz})$ \\
\hline Transmitted power $\left(P_{t}\right)$ & $30 \mathrm{dBm}$ & $30 \mathrm{dBm}$ \\
Transmitter antenna gain $\left(G_{t}\right)$ & $6 \mathrm{~dB}$ & $9 \mathrm{~dB}$ \\
Loss in free space $\left(L_{f}\right)$ & $28 \mathrm{~dB}($ in $25 \mathrm{~cm})$ & $35.6 \mathrm{~dB}($ in $25 \mathrm{~cm})$ \\
& $34 \mathrm{~dB}($ in $0.5 \mathrm{~m})$ & $41.68 \mathrm{~dB}($ in $0.5 \mathrm{~m})$ \\
Receiver antenna gain $\left(G_{r}\right)$ & $3.2 \mathrm{~dB}$ & $4.7 \mathrm{~dB}$ \\
Loss in body tissues $\left(L_{\text {implant }}\right)[22]$ & $15 \mathrm{~dB}$ & $22 \mathrm{~dB}$ \\
Received power by antenna in IPG $\left(P_{r}\right)$ & $0.41 \mathrm{~mW}$ (in $25 \mathrm{~cm})$ & $0.04 \mathrm{~mW}($ in $25 \mathrm{~cm})$ \\
& $0.1 \mathrm{~mW}$ (in $0.5 \mathrm{~m})$ & $0.01 \mathrm{~mW}($ in $0.5 \mathrm{~m})$ \\
\hline
\end{tabular}

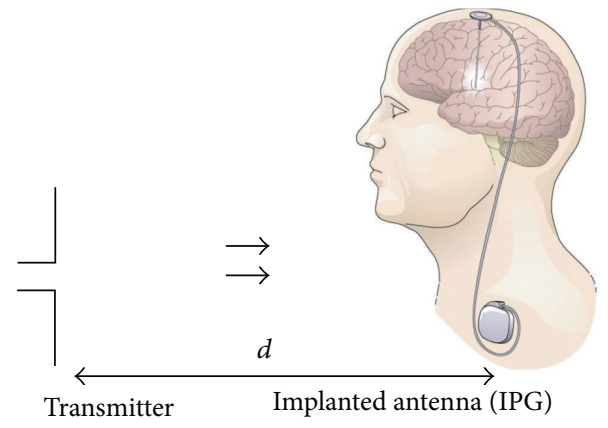

FIgURE 7: The layout of communication link between the external transmitter and the implanted receiving antenna for the energy harvesting performance evaluation.

a WIFI or WiMAX dual polarized transmitter to empower the DBS devices. Therefore, the energy harvesting efficiency using the antenna needs to be evaluated. To investigate the efficiency of the wireless link between the designed implanted antenna and the external transmitter, we consider the operational layout in Figure 7 for performance evaluation. In this setup, the communication link is established between the implanted antenna and the external transmitter. The harvesting efficiency of the antenna is evaluated based on the power received by the antenna. Using the Friis transmission theory, one can find the power received by the implanted antenna in the chest tissues as

$$
P_{r}(\mathrm{~dB})=P_{t}+G_{t}+G_{r}-L_{\text {implant }}-L_{f} \text {, }
$$

where $P_{r}$ is the received power by the implant antenna, $P_{t}$ is the transmitter power, $G_{t}$ is the transmitter gain, $G_{r}$ is the implant antenna gain, $L_{\text {implant }}$ is the power loss due to the dielectric of human body tissues, and $L_{f}$ is the power loss due to propagation in the free space and given by

$$
L_{f}=10 \log _{10}\left(\frac{4 \pi d}{\lambda}\right)^{2}
$$

The distances between the transmitter and the implant antenna are selected for the harvesting performance evaluation to be $0.5 \mathrm{~m}$ and $0.25 \mathrm{~m}$, and the simulations are performed to the test system at $2.4 \mathrm{GHz}$ and $5.8 \mathrm{GHz}$. Other relevant parameters used in the simulation are listed in Table 2.
The powers received by the implant antenna power at the distances of $0.25 \mathrm{~m}$ and $0.5 \mathrm{~m}$ at $2.4 \mathrm{GHz}$ and $5.8 \mathrm{GHz}$ are found to be $0.4 \mathrm{~mW}, 0.1 \mathrm{~mW}, 0.04 \mathrm{~mW}$, and $0.01 \mathrm{~mW}$.

The average power of maximum therapeutic energy is 2.8 to $5.4 \mathrm{~mW}$, but therapeutic energy level is variant and starts from $120 \mu \mathrm{W}$ [19]. In this work, the summation of harvested energy at both frequencies will be used by Rectenna. Hence, the received powers are sufficient for empowering the operation of the typical DBS implants.

In order to increase the harvested power for the implanted devices, one can choose to increase the transmission power, reduce the link distance, or harvest at a lower frequency to decrease the tissue loss.

\section{Conclusion}

An innovative approach is introduced to design modified Minkowski fractal patch antenna for efficient RF energy harvesting in dual ISM frequency bands for DBS applications. The simulation results show that the designed antenna can operate with two different resonant frequencies at $2.4 \mathrm{GHz}$ and $5.8 \mathrm{GHz}$ for efficient RF energy harvesting. The designed antenna is well matched at $2.4 \mathrm{GHz}$ with $-14 \mathrm{~dB}$ return loss and $3.2 \mathrm{dBi}$ gain and at $5.8 \mathrm{GHz}$ with $-11 \mathrm{~dB}$ return loss and $4.7 \mathrm{dBi}$ gain. The averaged SAR of the antenna in body tissue is found to be well below the legally allowed limit at both frequency bands and thus it is medically safe for implanting applications. The calculated link budget implies the designed antenna is capable of harvesting RF energy from external wireless radiation sources to empower the operation of passive DBS devices. In addition, the designed antenna can achieve significant physical miniaturization compared with the implantable antennas reported previously $[20,21]$.

\section{Conflict of Interests}

The authors declare that there is no conflict of interests regarding the publication of this paper.

\section{References}

[1] J. S. Perlmutter and J. W. Mink, "Deep brain stimulation," Annual Review of Neuroscience, vol. 29, pp. 229-257, 2006.

[2] A. L. Benabid, S. Chabardes, J. Mitrofanis, and P. Pollak, "Deep brain stimulation of the subthalamic nucleus for the treatment 
of Parkinson's disease," The Lancet Neurology, vol. 8, no. 1, pp. 67-81, 2009.

[3] C. R. Butson and C. C. McIntyre, "Differences among implanted pulse generator waveforms cause variations in the neural response to deep brain stimulation," Clinical Neurophysiology, vol. 118, no. 8, pp. 1889-1894, 2007.

[4] R. Merkel, Intervening in the Brain, Changing Psyche and Society, Ethics of Science and Technology Assessment, Springer, New York, NY, USA, 2007.

[5] M. T. Penella, "Powering wireless sensor nodes: Primary batteries versus energy harvesting," in Proceedings of the IEEE Instrumentation and Measurement Technology Conference, 2009.

[6] A. Kiourti, "Design of a novel miniaturized implantable PIFA for biomedical telemetry," in Proceedings of the 2nd International ICST Conference MobiHealth, pp. 127-134, Springer, 2010.

[7] D. G. Rucker, H. R. Khaleel, S. S. Raheem, and H. M. AlRizzo, "Microstrip antenna arrays for implantable and wearable wireless applications," in Wireless Mobile Communication and Healthcare, Proceedings of the 2nd International ICST Conference MobiHealth, pp. 135-143, Springer, Berlin, Germany.

[8] M. K. Hosain, A. Kouzani, and S. Tye, "Multi-layer implantable antenna for closed loop deep brain stimulation system," in Proceedings of the International Symposium on Communications and Information Technologies (ISCIT '12), pp. 643-648, October 2012.

[9] L. M. Li, H. W. Hao, B. Z. Ma, X. W. Wen, F. J. Liu, and C. $\mathrm{H}$. Hu, "Development of implantable neuro-stimulator used for brain and nerve stimulation," in 7th Asian-Pacific Conference on Medical and Biological Engineering IFMBE Proceedings, vol. 19, pp. 430-433, Springer, Berlin, Germany, 2008.

[10] C. A. Balanis, Antenna Theory Analysis and Design, Wiley, Hoboken, NJ, USA, 3rd edition, 2005.

[11] H. Xie, Fractals in Rock Mechanics, Geomechanics Research Series, chapter 2, CRC Press, Boca Raton, Fla, USA, 1993.

[12] Y.-W. Jang, "Experimental study of large bandwidth three-offset microstripline-fed slot antenna," IEEE Microwave and Wireless Components Letters, vol. 11, no. 10, pp. 425-427, 2001.

[13] J. Kim and Y. Rahmat-Samii, "Implanted antennas inside a human body: simulations, designs, and characterizations," IEEE Transactions on Microwave Theory and Techniques, vol. 52, no. 8, pp. 1934-1943, 2004.

[14] P. A. Hasgall, E. Neufeld, M. C. Gosselin, A. Klingenb, N. Kuster, and A. Klingenböck, "IT IS Database for thermal and electromagnetic parameters of biological tissues," 2013, http:// www.itis.ethz.ch/itis-for-health/tissue-properties/database/dielectric-properties/.

[15] http://www.fcc.gov/encyclopedia/specific-absorption-rate-sarcellular-telephones.

[16] http://www.clippercontrols.com/pages/Dielectric-ConstantValues.html.

[17] http://www.eccosorb.com.hk/sites/default/files/related_info/ Dielectric\%20Material\%20Chart.pdf.

[18] C. R. Butson, C. B. Maks, and C. C. McIntyre, "Sources and effects of electrode impedance during deep brain stimulation," Clinical Neurophysiology, vol. 117, no. 2, pp. 447-454, 2006.

[19] M. Belleville and C. Condemine, Energy Autonomous Micro and Nano Systems, chapter 2, Wiley, New York, NY, USA, 2012.

[20] P. Deepa, R. A. Sikkandhar, H. R. Suresh, and V. Naidu, "Designing a dual ISM band implantable antenna for medical monitoring applications using Dy-Sm doped magnesium Nano Ferrite material," International Journal of Computer Applications, vol. 44, no. 12, pp. 1-4, 2012.
[21] S. A. Kumar and T. Shanmuganantham, "Implanted CPW fed monopole antenna for biomedical applications," in Advances in Computing and Information Technology, vol. 178 of Advances in Intelligent Systems and Computing, pp. 97-105, 2013.

[22] K. Y. Yazdandoost and K. Sayrafian-Pour, "Channel model for body area network (BAN),” Tech. Rep. IEEE P802.15, Wireless Personal Area Networks, 2009. 

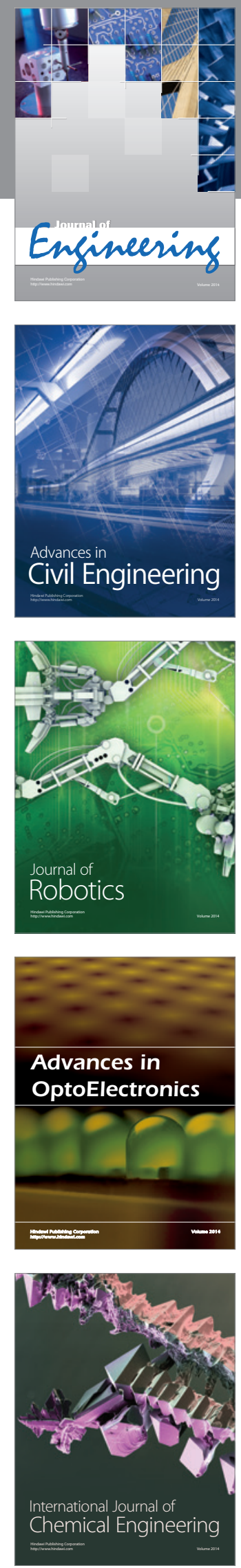

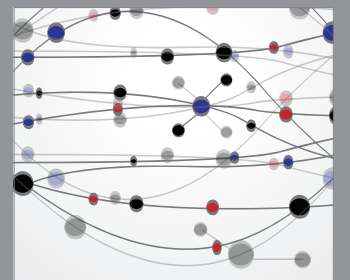

The Scientific World Journal
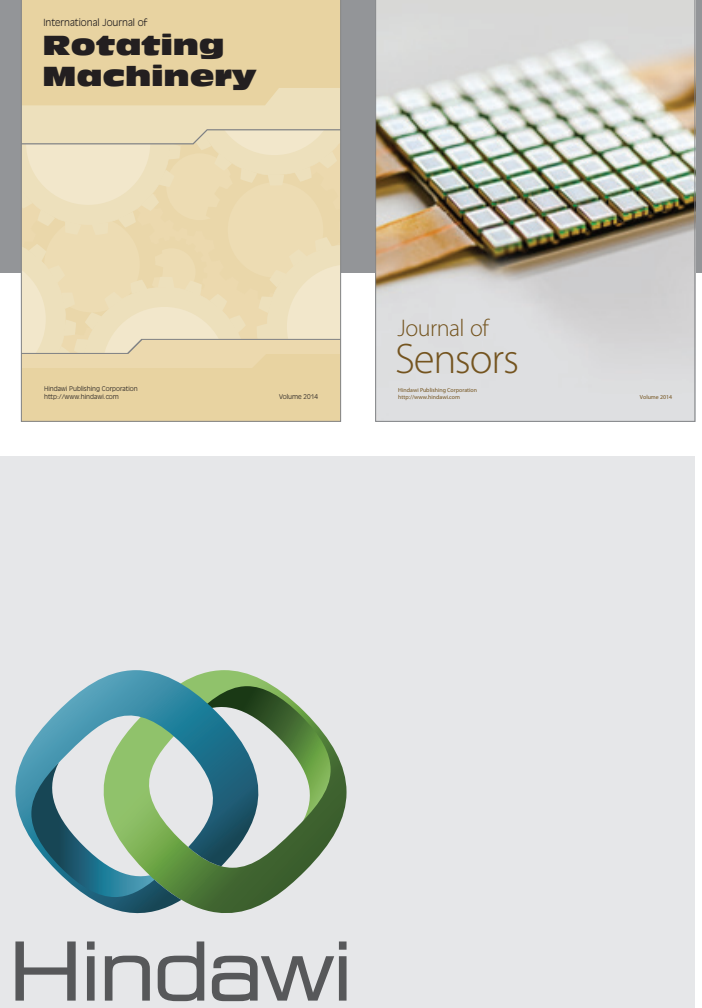

Submit your manuscripts at http://www.hindawi.com
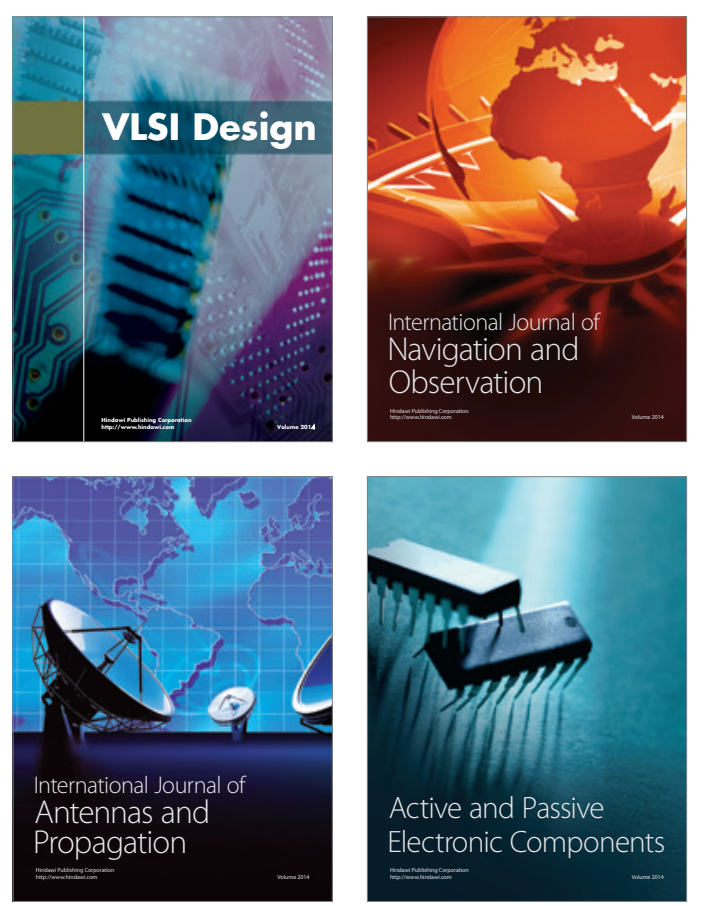
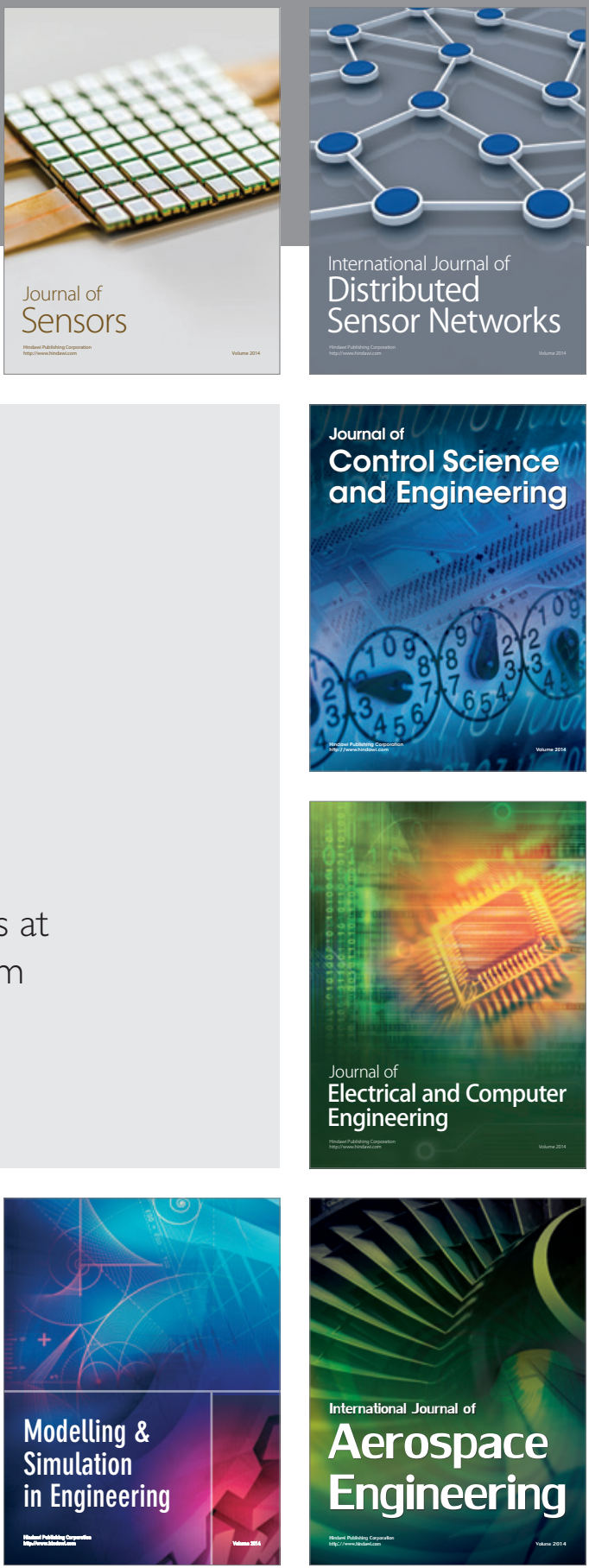

Journal of

Control Science

and Engineering
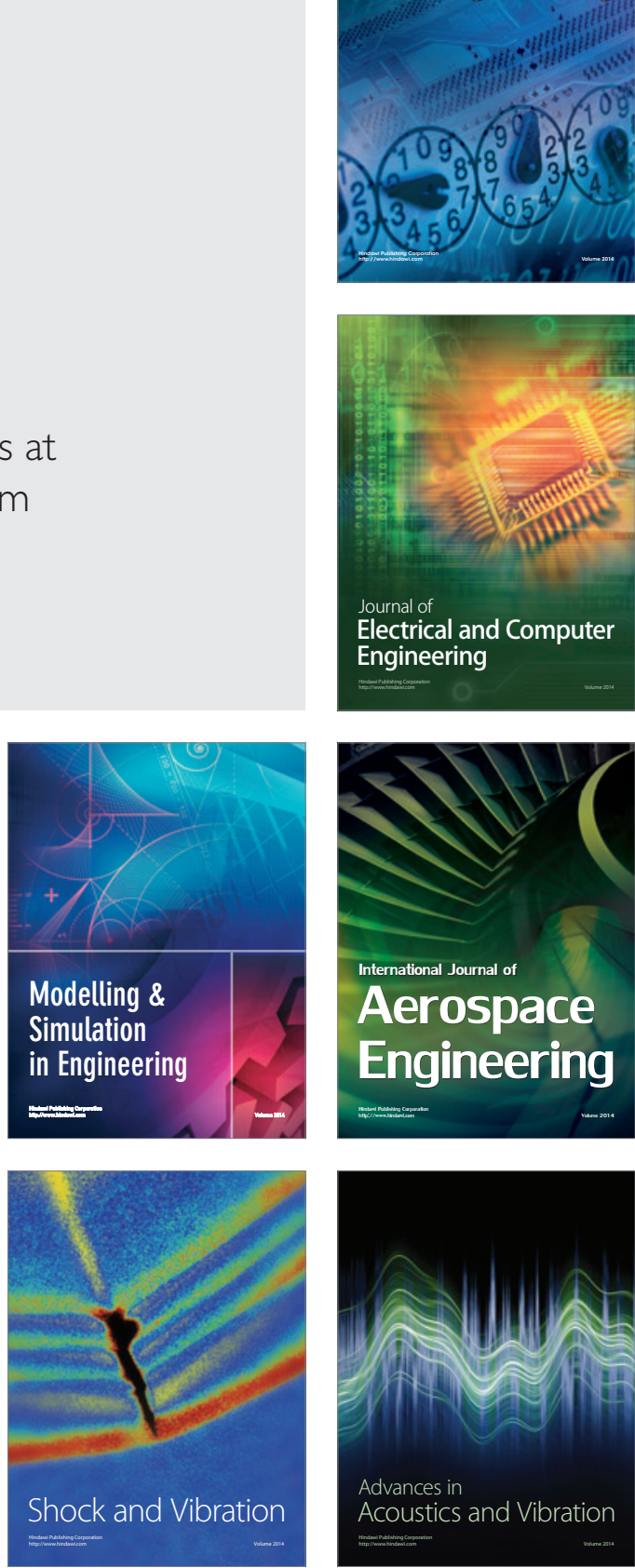